\section{$\underset{\substack{\text { hommes } \\ \text { \& migrations }}}{ }$}

\section{Hommes \& migrations}

Revue française de référence sur les dynamiques

migratoires

$1285 \mid 2010$

L'appel du pied

\title{
L'internationalisation du marché des footballeurs
}

Le cas français (1960-2010)

\section{Raffaele Poli et Loïc Ravenel}

\section{OpenEdition}

1 Journals

\section{Édition électronique}

URL : http://journals.openedition.org/hommesmigrations/1175

DOI : 10.4000/hommesmigrations. 1175

ISSN : 2262-3353

Éditeur

Musée national de l'histoire de l'immigration

Édition imprimée

Date de publication : 1 mai 2010

Pagination : 48-57

ISSN : 1142-852X

\section{Référence électronique}

Raffaele Poli et Loïc Ravenel, «L'internationalisation du marché des footballeurs », Hommes \& migrations [En ligne], 1285 | 2010, mis en ligne le 29 mai 2013, consulté le 01 mai 2019. URL : http:// journals.openedition.org/hommesmigrations/1175; DOI : 10.4000/hommesmigrations. 1175 


\section{L'internationalisation du marché des footballeurs \\ Le cas françails (1960-2010)}

Par Raffaele Poli, Institut des sciences du sport (université de Lausanne); Centre international d'étude du Sport (université de Neuchâtel) et Loïc Ravenel, Laboratoire ThéMA (université de Franche-Comté)

Du début des années trente à nos jours, le recrutement international des clubs français de Ligue 1 a dû compter avec les dispositifs juridiques encadrant l'entrée des joueurs non nationaux dans l'Hexagone. À l'heure actuelle, la disparition des quotas draine un nombre important de joueurs expatriés provenant en grande partie du continent africain et d'Amérique latine. Pays importateur de longue date, la France alimente également les grands championnats européens grâce à la qualité de ses structures de formation. 
Limportation de footballeurs professionnels en France remonte au moins à l'origine de la Ligue de football professionnel (1932). Selon Pierre Lanfranchi, entre 1932 et 1939, les clubs français de première division ont employé 329 footballeurs étrangers, dont un grand nombre depuis l'ancienne Yougoslavie ${ }^{(1)}$. L'importation de joueurs est néanmoins restée pendant longtemps limitée par l'existence de quotas stricts sur l'emploi de non nationaux. Avant 1960, de nombreux joueurs ont été importés depuis les anciennes colonies africaines, en particulier l'Algérie ${ }^{(2)}$. Ces footballeurs étaient alors considérés comme des nationaux et n'étaient de ce fait pas soumis à des quotas. Cette situation a changé après les indépendances des pays anciennement colonisés. Cet article se concentre sur la période qui s'étend de 1960 à 2010.

\section{L'importation de joueurs}

Entre 1960 et 1985, le nombre de footballeurs importés présents dans les équipes du premier niveau de compétition français est relativement stable. Certes, la présence d'expatriés est plus forte dans les années soixante-dix que dans les années soixante, mais le nombre moyen de ces joueurs par club ne dépasse jamais les trois unités (graphique 1). Ce résultat est directement lié à l'existence de quotas qui limitent drastiquement la présence de joueurs ne possédant pas de passeport français. La situation change à partir de la fin des années quatre-vingt, lorsque le nombre de footballeurs non nationaux tolérés passe à cinq : trois "étrangers" et deux "assimilés". Ces derniers sont des joueurs étrangers présents en France depuis au moins cinq saisons (cf. graphique 1).

Une autre décision juridique explique la forte augmentation observée après 1995 : l'arrêt Bosman de la Cour de justice des Communautés européennes. Cette sentence a aboli les quotas jusque-là appliqués pour les footballeurs détenant un passeport communautaire ${ }^{(3)}$. Depuis cette date, un ressortissant d'un pays membre de l'Union européenne est considéré comme national et bénéficie à ce titre de la libre circulation au sens des articles 48 et 49 du Traité instituant la Communauté économique européenne (Traité de Rome du 25 mars 1957).

Fin 2002, l'arrêt Malaja émis par le Conseil d'État français pousse la Ligue de football professionnel française à élargir encore davantage le cercle des pays dont les ressortissants ne sont plus soumis à des quotas. Désormais, tout joueur originaire d'une centaine de pays extérieurs à l'Union européenne, dont beaucoup d'anciennes colonies françaises, n'est plus comptabilisé dans les quotas limitant le nombre de non-communautaires, dans la mesure où il a déjà disputé un match dans la sélection nationale de son pays de provenance, même au niveau junior ${ }^{(4)}$. 
Graphique 1 : Nombre de joueurs expatriés par club, Ligue 1 (1960-2010)

Grâce à ces changements réglementaires, les clubs français ont pu importer un nombre inégalé de footballeurs. Lors de la saison 2005-2006, les équipes de Ligue 1 comptaient dans leurs rangs en moyenne 9,6 joueurs arrivés en France dans le cadre d'un recrutement effectué en lien direct avec le football. Les expatriés représentent alors 34,6\% de l'ensemble des footballeurs employés par les clubs de Ligue 1, un niveau jamais atteint par le passé. Depuis 2005, le nombre et le pourcentage de joueurs expatriés par club s'est stabilisé. Lors de la saison 20092010, ils sont en moyenne 9,2 par club, soit 31,8 \% des effectifs.

\section{La France comparée aux quatre grandes ligues européennes}

Jusqu'en 1990, les clubs français emploient un nombre de joueurs expatriés relativement plus élevé que les équipes des quatre autres grandes ligues européennes (graphique 2). Ce résultat peut s'expliquer par la combinaison de différents facteurs : un régime de quotas parfois moins strict qu'ailleurs, des critères d'acquisition de la nationalité moins restrictifs, ou encore une plus grande ouverture au monde héritée du passé colonial. À partir de 1995, la proportion de 
footballeurs arrivés dans l'Hexagone pour exercer leur métier devient similaire à celle observée ailleurs, voire plus faible. Ainsi, lors de la saison 2009-2010, l'indice de présence d'expatriés dans les équipes françaises par rapport aux quatre autres grandes ligues européennes n'est plus que de 0,7 , un minimum historique.

\section{Graphique 2 : Rapport entre le nombre d'expatriés par club en France et celui dans les quatre autres grandes ligues européennes (Angleterre, Allemagne, Espagne, Italie) (1960-2010)}

Tableau 1 : Pourcentage de joueurs expatriés dans l'effectif (2005-2010)

\begin{tabular}{|l|c|c|c|}
\hline CHAMPIONNAT & $\begin{array}{c}\text { \% expatriés } \\
(2005-06)\end{array}$ & $\begin{array}{c}\text { \% expatriés } \\
(2009-10)\end{array}$ & Écart \\
\hline Angleterre & 55,2 & 59,8 & $+4,6$ \\
\hline Allemagne & 40,8 & 48,0 & $+7,2$ \\
\hline Italie & 30,5 & 42,2 & $+11,7$ \\
\hline Espagne & 31,6 & 32,7 & $+1,1$ \\
\hline France & 34,6 & 31,8 & $-2,8$ \\
\hline
\end{tabular}




\section{Tableau 2 : Les filux de joueurs entre les cinq grandes ligues européennes (2005-2010)}

\begin{tabular}{|c|c|c|c|c|c|c|}
\hline $\begin{array}{l}\text { ORIGINE/ } \\
\text { DESTINATION }\end{array}$ & Angleterre & Espagne & France & Allemagne & Italie & Départs \\
\hline Angleterre & & 51 & 31 & 19 & 28 & 129 \\
\hline Espagne & 54 & & 22 & 13 & 47 & 136 \\
\hline France & 67 & 29 & & 28 & 16 & 140 \\
\hline Allemagne & 34 & 18 & 15 & & 11 & 78 \\
\hline Italie & 33 & 40 & 17 & 13 & & 103 \\
\hline Arrivées & 188 & 138 & 85 & 73 & 102 & 586 \\
\hline
\end{tabular}

La particularité de la France apparaît encore avec la prise en compte des pays de manière individuelle (tableau 1). Les chiffres montrent d'importants écarts entre pays latins et pays non latins, les premiers employant significativement moins de footballeurs expatriés que les seconds. Entre 2005 et 2010, le pourcentage d'expatriés a augmenté partout sauf en France, où les clubs réunissent désormais en moyenne le plus faible nombre de joueurs importés. Cette situation reflète à la fois l'excellence du système de formation français, qui agit positivement sur l'offre locale de talents, et la relative paupérisation des clubs hexagonaux vis-à-vis notamment des équipes anglaises et des meilleurs clubs espagnols.

Ce double constat peut aussi se lire en analysant la balance des flux internationaux intervenus entre les équipes des cinq principales ligues européennes entre la saison 2005-2006 et la saison 2009-2010 (tableau 2).

Entre 2005 et 2010, les clubs de Ligue 1 française ont cédé 140 joueurs à des équipes des quatre autres grandes ligues européennes. Seuls 85 footballeurs ont par contre parcouru le chemin opposé. À l'inverse, en Angleterre, le bilan est très positif avec 188 joueurs importés contre 129 exportés $^{(5)}$. Néanmoins, si la France est exportatrice vis-à-vis des autres puissances européennes, elle reste un pays importateur à l'échelle mondiale.

\section{L’origine des expatriés en France}

Afin de faciliter la lecture des données, l'analyse des origines des joueurs importés se base sur le regroupement des pays dans quatre grandes zones géographiques: l'Europe de l'Ouest, l'Europe de l'Est, l'Amérique latine et l'Afrique. L'Europe de 
l'Ouest comprend l'ensemble des pays de la partie occidentale du continent, ainsi que la Grèce. L'Europe de l'Est réunit tous les pays ayant fait partie du bloc soviétique. L'Amérique latine regroupe les pays anciennement colonisés par l'Espagne et le Portugal. L'Afrique comprend tous les pays de ce continent, y compris ceux du Maghreb. De ces quatre zones sont presque exportés la totalité des joueurs expatriés présents dans les clubs français.

\section{Graphique 3 : Évolution de la répartition des footballeurs expatriés en France par zone d’origine (1960-2010)}

À l'aube des indépendances, plus de la moitié des footballeurs expatriés présents dans les clubs de Ligue 1 française proviennent d'Afrique, tant du Maghreb (du Maroc principalement) que d'Afrique subsaharienne (surtout du Cameroun). À cela s'ajoutent des joueurs d'autres nations d'Europe de l'Ouest (surtout des PaysBas et d'Autriche) et d'Amérique latine (surtout d'Argentine), alors que les joueurs d'Europe de l'Est sont presque absents. Au total, 16 pays sont représentés.

Lors de la décennie qui suit les indépendances, l'importation de joueurs africains est d'abord remplacée par des recrutements effectués en Amérique latine (en Argentine et au Paraguay), puis en Europe de l'Est (en ancienne Yougoslavie pour la plupart) et en Europe de l'Ouest (surtout au Danemark et, dans une moindre mesure, en Suède). En 1970, les ressortissants européens représentent 80,4 \% des expatriés, contre 30,3\% dix saisons auparavant. La part des Africains a dans le 
même temps diminué de 54,5 à 14,3\%. Ces chiffres reflètent l'existence d'un processus de substitution ayant fait suite aux indépendances. Lors de la saison 197071,21 pays sont représentés en première division française.

La présence de footballeurs importés depuis l'Europe de l'Est reste longtemps prédominante. Entre 1970 et 1985, ces footballeurs représentent toujours au moins 31 \% des effectifs d'expatriés, avec au final presque autant de Polonais que de Yougoslaves. Durant cette période, nous observons également une reprise des recrutements en Amérique latine d'abord, puis en Afrique. Ces derniers s'effectuent surtout au détriment des footballeurs d'autres pays d'Europe occidentale. En 1985, le pourcentage de joueurs africains (principalement du Cameroun et de la Côte d'Ivoire) et latino-américains (surtout d'Argentine) parmi les expatriés remonte à 50 \%. Le nombre de pays représentés est de 19, deux unités de moins qu'en 1970. La période entre 1985 et 1995 se caractérise par deux étapes distinctes. Lors des cinq premières années, la présence relative de footballeurs d'Europe de l'Ouest (surtout des Pays-Bas et du Danemark) augmente au détriment de celle des joueurs africains et latino-américains. On observe par contre une tendance opposée entre 1990 et 1995, lorsque la part des Africains parmi les expatriés s'accroît de nouveau fortement. Malgré la chute du mur de Berlin, la part de joueurs d'Europe de l'Est continue de diminuer, en passant de 31 \% en 1985 à 26,2 \% en 1995. À la veille de l'arrêt Bosman, les footballeurs expatriés en France proviennent de 33 pays, un nombre jamais atteint par le passé reflétant l'augmentation générale des importations.

\section{Les effets de l'élargissement des quotas}

La décennie de 1995 à 2005 peut aussi être divisée en deux étapes. Dans un premier temps, la part des joueurs africains et d'Europe de l'Est diminue au profit des footballeurs d'Europe de l'Ouest (surtout de Belgique) et, plus encore, d'Amérique latine (surtout d'Argentine et de Brésil). Cette situation s'explique par le fait que le nouveau régime juridique régissant les migrations internationales des footballeurs favorise les joueurs possédant un passeport communautaire. Les footballeurs d'Amérique latine détiennent en ce sens un avantage dans la mesure où ils peuvent souvent faire valoir des origines européennes et acquérir de ce fait un passeport communautaire.

L'abandon progressif des quotas limitant le nombre de joueurs non communautaires à partir de l'arrêt Malaja de 2002 explique le nouveau renversement de tendance intervenu entre 2000 et 2005 en ce qui concerne les Africains. Leur proportion parmi 
les expatriés passe de 33,1 \% à 46,1\%. L'augmentation la plus notable concerne les Sénégalais et les Ivoiriens. Entre 2000 et 2005, alors que la part de footballeurs d'Amérique latine (surtout du Brésil) continue de s'accroître, celle des joueurs d'Europe de l'Est et d'Europe de l'Ouest diminue sensiblement. Lors de la saison 2005-06, 71,8 \% des footballeurs expatriés proviennent d'un pays situé en dehors de l'Europe, un pourcentage qui n'avait plus été aussi élevé depuis 1965. En 2005, le nombre de pays représentés atteint une nouvelle valeur maximale de 41 .

Les données les plus récentes (saison 2009-2010) montrent que l'importation de footballeurs en

\section{La France a été l'un des premiers pays en Europe à mettre en place un système de formation de footballeurs.} France se fait toujours prioritairement depuis des pays non européens. Les joueurs extra-européens représentent 66,7 \% des expatriés, avec une grande stabilité au niveau des LatinoAméricains (25,7\% des effectifs) et une diminution relative des Africains (-5,1\% par rapport à 2005). Si l'Afrique reste la principale zone d'origine des expatriés, nous observons leur substitution partielle par des footballeurs d'autres origines. Cette tendance peut s'expliquer par les plus fortes restrictions intervenues au niveau du transfert de mineurs, une pratique jusque-là courante, surtout depuis l'Afrique. Elle peut aussi être interprétée comme le reflet d'une diversification des réseaux de recrutement mobilisés par les clubs français dans le contexte de la mondialisation du marché des footballeurs ${ }^{(6)}$.

\section{Les Français à l'étranger}

La France a été l'un des premiers pays en Europe à mettre en place un système de formation de footballeurs. En 1973, les clubs professionnels ont dû se doter de structures assurant une formation de très haut niveau. Si cette obligation a été levée en 2003, les équipes françaises restent des références en matière de formation. Le savoir-faire acquis par les expériences passées a permis de créer un avantage compétitif durable.

Lors de la saison 2009-2010, avec 106 joueurs expatriés, la France se classe à la troisième place des pays ayant le plus grand nombre de footballeurs dans les quatre autres grandes ligues européennes, juste derrière le Brésil (139, dont 23 en France) et l'Argentine (120, dont 15 en France). Les Français constituent la première nationalité étrangère représentée en Angleterre (51 joueurs), la deuxième en Allemagne (19), ainsi que la troisième en Espagne (22) et en Italie (16). 
Tableau 3 : Les dix principaux pays exportateurs dans 36 ligues de première division de pays membres de IUUFA, octobre 2009

\begin{tabular}{|l|c|c|}
\hline PAYS & Nombre d'expatriés & Pourcentage cumulé \\
\hline Brésil & 502 & 12,3 \\
\hline France & 239 & 18,1 \\
\hline Argentine & 238 & 23,9 \\
\hline Serbie & 201 & 28,8 \\
\hline Portugal & 129 & 32,0 \\
\hline Tchéquie & 118 & 34,8 \\
\hline Nigeria & 113 & 37,6 \\
\hline Angleterre & 102 & 40,1 \\
\hline Allemagne & 97 & 42,5 \\
\hline Suède & 97 & 44,8 \\
\hline
\end{tabular}

Limportance de la France en tant que pays de formation et d'exportation de footballeurs peut aussi se lire à plus vaste échelle par l'analyse de la présence de joueurs expatriés dans 36 ligues de première division de pays membres de l'UEFA. Selon le recensement effectué au mois d'octobre 2009, il y avait alors 239 joueurs français à l'étranger, ce qui situe la France en deuxième position, derrière le Brésil et devant l'Argentine (tableau 3) ${ }^{(7)}$.

L'analyse des clubs dans lesquels évoluent les joueurs sélectionnés par Raymond Domenech pour la phase finale de la Coupe du monde 2010 confirme cette tendance à l'exportation des joueurs français. Parmi les 23 convoqués, 12 jouent dans des clubs étrangers, dont 7 en Angleterre et 3 en Espagne. À titre de comparaison, aucun joueur des équipes italienne et anglaise n'évolue dans des clubs étrangers.

\section{Conclusion}

Entre 1960 et 2010, nous observons une augmentation générale de l'importation de footballeurs en France, étroitement liée à l'affaiblissement des limites juridiques ayant longtemps maintenu les flux internationaux à un niveau artificiellement bas. Comme dans les années soixante, les joueurs expatriés proviennent toujours principalement des pays africains (surtout du Cameroun, du Sénégal, de la Côte d'Ivoire et du Mali) et latino-américains (Brésil et Argentine). 
Depuis une quinzaine d'années, nous observons également une forte exportation de footballeurs de la France vers les autres grands championnats européens. Ces flux interviennent dans le contexte d'un processus de polarisation économique qui favorise notamment les clubs anglais. La France tend ainsi à devenir un espace tremplin destiné à lancer des talents locaux, souvent issus des milieux défavorisés de la société, et à accueillir des jeunes joueurs importés depuis des pays éloignés dans l'optique d'un transfert vers des clubs plus riches, dont beaucoup se situent Outre-manche.

Le plus faible taux d'expatriés dans les clubs français par rapport aux équipes des autres principales ligues européennes témoigne de l'excellence du système de formation mis en place depuis les années soixante-dix, mais aussi des difficultés rencontrées par les clubs hexagonaux pour garder leurs meilleurs éléments dans un contexte économique qui leur est relativement défavorable.

\section{Bibliographie}

- R. Besson, R. Poli, et L. Ravenel, Étude démographique des footballeurs en Europe, Neuchâtel, CIES, 2010.

- J.-P. Dubey, La Libre Circulation des sportifs en Europe, Berne, Stämpfli, 2000.

- S. Frenkiel et N. Bancel, "The Migration of Professional Algerian Footballers to the French Championship, 1956-82: The 'Desire for France' and the Prevailing National Contexts", International Journal of the History of Sport, vol. 25, $\mathrm{n}^{\circ} 8$, 2008, pp. 1031-1050.

- P. Lanfranchi, "The Migration of Footballers: The Case of France, 1932-1982", in J. Bale et J. Maguire, The Global Sports Arena: Athletic Talent Migration in an Interdependent World, London, Frank Cass, 1994, pp. 63-77.

- R.Poli, "Les politiques migratoires dans le football européen. Quotas et naturalisations dans une optique géopolitique", Histoire et Sociétés, n 18-19, pp. 46-61.

- R. Poli, Le Marché mondial des footballeurs, Berne, Peter Lang, 2010.

- R. Poli, L. Ravenel, et R. Besson, Étude annuelle du marché du travail européen des footballeurs, Neuchâtel, CIES, 2009.

\section{Notes}

1. P. Lanfranchi, "The Migration of Footballers: The Case of France, 1932-1982”, in J. Bale et J. Maguire, The Global Sports Arena: Athletic Talent Migration in an Interdependent World, London, Frank Cass, 1994, pp. 63-77.

Les données utilisées dans cet article ont été collectées par le recoupement d'une multitude de sources dans le cadre des activités de l'Observatoire des footballeurs professionnels. Il s'agit d'un groupe de recherche franco-suisse créé en 2005 réunissant des géographes des universités de Lausanne, de Neuchâtel et de Franche-Comté. Plus d'informations sous www.eurofootplayers.org

2. S. Frenkiel et N. Bancel, "The Migration of Professional Algerian Footballers to the French Championship, 1956-82: The 'Desire for France' and the Prevailing National Contexts", International Journal of the History of Sport, vol. 25, $\mathrm{n}^{\circ} 8$, 2008, pp. 1031-1050.

3. J.-P. Dubey, La Libre Circulation des sportifs en Europe, Berne, Stämpfli, 2000.

4. R.Poli, "Les politiques migratoires dans le football européen. Quotas et naturalisations dans une optique géopolitique", Histoire et Sociétés, n 18-19, pp. 46-61.

5. R. Poli, L. Ravenel, et R. Besson, Étude annuelle du marché du travail européen des footballeurs, Neuchâtel, CIES, 2010.

6. R. Poli, Le Marché mondial des footballeurs, Berne, Peter Lang, 2010.

7. R. Besson, R. Poli, et L. Ravenel, Étude démographique des footballeurs en Europe, Neuchâtel, CIES, 2010. 